\title{
Estimation of three-dimensional volumetric errors of machining centers by a tracking interferometer
}

\author{
$\operatorname{AUTHOR}(\mathrm{S})$ : \\ Ibaraki, Soichi; Kudo, Tomoya; Yano, Tomoaki; \\ Takatsuji, Toshiyuki; Osawa, Sonko; Sato, Osamu
}

\section{CITATION:}

Ibaraki, Soichi ... [et al]. Estimation of three-dimensional volumetric errors of machining centers by a tracking interferometer. Precision Engineering 2015, 39: 179-186

\section{ISSUE DATE:}

2015-01

URL:

http://hdl.handle.net/2433/192479

\section{RIGHT:}

C 2014 Elsevier Inc.; この論文は出版社版でありません。引用の際には 出版社版をご確認ご利用ください。; This is not the published version. Please cite only the published version. 


\title{
Estimation of Three-dimensional Volumetric Errors of Machining Centers by a Tracking Interferometer
}

\author{
Soichi Ibaraki ${ }^{\mathrm{a}}$, Tomoya Kudo ${ }^{\mathrm{a}}$, Tomoaki Yano ${ }^{\mathrm{b}}$, Toshiyuki Takatsujic, \\ Sonko Osawa ${ }^{c}$, Osamu Sato ${ }^{c}$ \\ ${ }^{a}$ Department of Micro Engineering, Kyoto University, \\ Yoshida-honmachi, Sakyo-ku, Kyoto 606-8501, Japan. Phone/Fax: +81-75-753-5227 \\ ${ }^{b}$ Tomoaki Yano, Faculty of Engineering, Kinki University, Japan. \\ ${ }^{c}$ National Metrology Institute of Japan, National Institute of Advanced Industrial Science \\ and Technology, Japan.
}

\begin{abstract}
A tracking interferometer is a laser interferometer with the mechanism to steer the laser direction to automatically follow a target retroreflector. This paper experimentally investigates the performance of the tracking interferometer prototype, developed by a part of the authors, in estimating the volumetric accuracy of a machining center based on the multilateration principle. Then, the prototype's technical issues are discussed based on the measurement uncertainty analysis. This paper briefly reviews the direct algorithm to calculate the three-dimensional position of the target, as well as the indirect algorithm to estimate geometric error parameters of the machine's kinematic model. Their comparison is also presented based on the uncertainty analysis. Keywords: Tracking interferometer, laser tracker, volumetric errors, multilateration, machine tools, kinematic model
\end{abstract}

Email address: ibaraki@prec.kyoto-u.ac.jp (Soichi Ibaraki) 


\section{Introduction}

ISO TC39/SC2, a technical subcommittee in International Organization of Standardization (ISO), has lately been discussing the publication of a Technical Report (TR) on numerical compensation for geometric errors in machine tools [1]. Such an effort clearly indicates the recognition by machine tool manufacturers/users of the importance of the machine tool volumetric accuracy. In ISO 230-1:2012 [2], recently revised by ISO TC39/SC2, the definition of the term "volumetric accuracy" is newly added, as well as a new annex describing the machine tool geometric error parameters. In the industry, many major CNC makers have lately commercialized the functionality of numerically compensating for volumetric errors.

On many commercial machine tool CNCs, numerical compensation for linear positioning error, caused typically by the pitch error of a ball screw or a linear encoder, is common. Some CNCs further compensate for the straightness or squareness error in linear axes. The volumetric error compensation is an extension of these simpler compensations to three-dimensional space. The objective of volumetric error compensation is to cancel an error in the three-dimensional tool center position (TCP) at an arbitrary point in the workspace by adjusting its command position in $\mathrm{X}-, \mathrm{Y}-$, and Z-directions. The volumetric error compensation has been more common in coordinate measuring machines (CMMs). Its application to machine tools has been long studied [3, 4].

Conventionally, as described in ISO 230-1 [2], the inspection of the motion accuracy of a machine tool is based on the axis-to-axis measurement; for each axis, various error motions, e.g. the linear positioning error motion, straight- 
ness errors, and angular error motionss, are measured independently. Based on a kinematic model, one can calculate the three-dimensional positioning error at an arbitrary position [5]. According to Schwenke et al. [4], such an evaluation of the volumetric accuracy is called "indirect" measurement of volumetric errors. On the other hand, "direct" measurement of volumetric errors mean direct measurement of the TCP at arbitrary locations. An established direct measurement method uses calibrated three-dimensional artifacts, e.g. three-dimensional ball plates. Such artifact-based calibrations are more common for CMMs (ISO 10360-2:2009 [6]). Its application to machine tools has been also reported [7]. For the application to large-sized machine tools, a large artifact of the required geometric accuracy is needed, which is often difficult and/or expensive.

The tracking interferometer (the term in [2]), or the laser tracker, is probably only commercially available instrument capable of measuring the threedimensional TCP at an arbitrary location within its workspace. It is a laser interferometer with a steering mechanism to change the laser beam direction to automatically follow a target retroreflector. Some commercial laser trackers, from, e.g., Leica Geosystems, Faro, and Automated Precision Inc. (API), measure the TCP from the distance (displacement) to the target and the direction of the laser beam [8]. Since its angular measurement uncertainty directly contributes to the target position's estimation uncertainty, it is typically difficult to ensure the measurement accuracy high enough to evaluate machine tools.

The application of tracking interferometers to the multilateration-based measurement, where the target's three-dimensional position is estimated by 
the distance (displacement) from typically four or more tracking interferometers to the target, has been studied for machine tool calibration [9, 10, 11]. Its commercial product has been recently introduced (Etalon AG [9, 12]). In the multilateration principle, the instrument's angular positioning uncertainty does not significantly contribute on the measurement uncertainty.

A part of the authors in National Institute of Advanced Industrial Science and Technology developed a tracking interferometer prototype. Its design consideration and laser measurement uncertainty analysis were presented in [13], but its performance in the multilateration measurement was not investigated. This paper presents experimental investigation of its performance in measuring three-dimensional positioning errors of a machining center. Based on its uncertainty analysis, the prototype's technical issue will be discussed.

This paper reviews 1) the direct algorithm to calculate the three-dimensional target position from tracking interferometer measurements, and 2) the indirect algorithm to estimate geometric error parameters of the machine's kinematic model. Following their experimental demonstration, their comparison from the viewpoint of the uncertainty propagation will be presented.

\section{Prototype tracking interferometer}

A part of the authors has developed a couple of different tracking interferometers. Earlier prototypes in $[14,15,16]$ used an XY-stage to control the orientation of a hemispheric mirror. In this study, we use the latest prototype tracking interferometer developed in [13] where a spherical motor [17] is employed to orientate a hemispheric mirror. Its outlook is shown in Fig. 1. Its major specifications are shown in Table 1. 
A retroreflector, referred to as the "target" in this paper, is attached to the machine's spindle. In this study, we use a cat's eye retroreflector as the target. The cat's eye retroreflector is a spherical glass of the pre-calibrated geometric accuracy with its hemispheric surface coated by the total-reflection metal-film deposition [18], so that the incoming light is reflected to the same direction. Its major specifications are shown in Table 2.

Figure 2 illustrates the tracking mechanism. When (a) the shift of the laser spot on the quadrant photo diode (QPD) is detected, (b) the mirror is tilted by a spherical motor (in two directions) to regulate the laser spot position on the QPD. Figure 3 illustrates the control system configuration. Signals from the QPD are transmitted to a PC-based real-time control system (LT-RTSim by DSP Technology), where the laser spot displacement is calculated. The spherical motor is controlled by a servo amplifier (SGDS-A5F05A by Yaskawa Electric Corp.). The current and velocity feedback loops are implemented in the servo amplifier, and the position feedback loop is programmed in the PC-based control system. A laser interferometer, DISTAX L-IH-302A by Tokyo Seimitsu Co., Ltd., with He-Ne laser (the vacuum wavelength $633.0 \mathrm{~nm}$ ), is used. The laser displacement is counted by a counter board, DISTAX LD-301 by Tokyo Seimitsu Co., Ltd., and logged on a PC. The influence of air temperature, pressure and humidity on the laser wavelength is compensated.

There are many potential uncertainty contributors associated with this tracking interferometer mechanism. Besides the contributors on laser length measurement uncertainties, e.g. environmental uncertainties, the radial error motion of the spherical motor directly contributes on the laser displacement. 
Note that, on the other hand, the angular positioning error of the spherical motor, caused by the QPD-based feedback control system, only contributes on the uncertainty in the laser beam direction. In principle, the laser beam direction error only gives the "cosine error" on the laser displacement, and thus its contribution is often sufficiently small. The uncertainty analysis for this prototype tracking interferometer was presented in [16].

\section{Direct measurement algorithm: an algorithm to estimate target position}

The objective of the direct measurement algorithm to calculate the target position from the laser displacement measured from tracking interferometers at different positions. The essential difference of this problem from the trilateration principle is that 1) the exact position of each tracking interferometer is not known, and 2) the absolute distance from the interferometer to the target cannot be measured (only the relative distance to the initial position can be measured by a laser interferometer). To solve this problem, a self-calibration approach by using a redundant measurement from more than three tracking interferometers has been well developed, e.g. [16]. This subsection only briefly reviews this algorithm.

Figure 4 illustrates the problem setup. The problem to calculate target positions, $p_{i} \in \mathbb{R}^{3}(i=1, \cdots, N)$, is given as the following minimization problem:

$$
\min _{x} \sum_{i=1 \cdots N, j=1 \cdots N_{t}}\left(f_{i j}(x)-d_{i j}\right)^{2}
$$

where $d_{i j} \in \mathbb{R}$ represents the laser displacement measured by the $j$-th tracking interferometer to the $i$-th target position. The function, $f_{i j}: \mathbb{R}^{3 N+4 N_{t}} \rightarrow \mathbb{R}$, is 
given by:

$$
f_{i j}(x)=\left\|p_{i}-P_{j}\right\|-d_{0 j}
$$

where $P_{j} \in \mathbb{R}^{3}\left(j=1, \cdots, N_{t}\right.$, where $\left.N_{t} \geq 4\right)$ represents the $j$-th tracking interferometer position. $d_{0 j} \in \mathbb{R}$ represents the dead path length in the measurement by $j$-th tracking interferometer [9]. $x \in \mathbb{R}^{3 N+4 N_{t}}$ represents a set of unknown parameters to be identified, containing:

$$
x=\left[\left\{p_{i}\right\}_{i=1, \cdots, N},\left\{P_{j}\right\}_{j=1, \cdots, N_{t}},\left\{d_{0 j}\right\}_{j=1, \cdots, N_{t}}\right] \in \mathbb{R}^{3 N+4 N_{t}}
$$

Note that total six parameters can be constrained by the coordinate system. For $3 N+4 N_{t}-6$ unknown parameters, the number of laser measurements is $N \cdot N_{t}$. Therefore, when $N>\frac{4 N_{t}-6}{N_{t}-3}$, the number of measurements exceeds the number of unknown parameters. Since the problem (1) is a nonconvex problem, an iterative linearization-based approach is typically used to locally solve it.

\section{Indirect measurement algorithm: an algorithm to estimate the machine tool's geometric error parameters}

Recently revised ISO 230-1:2012 [2] includes a new annex describing geometric error parameters of the machine's kinematic model. Geometric error parameters represent the position and the orientation of each linear or rotary axis at the given position. Schwenke et al. [9] presented an algorithm to estimate geometric error parameters from a set of measured laser displacements, $d_{i j}\left(i=1, \cdots, N, j=1, \cdots, N_{t}\right)$. This section briefly reviews it.

The kinematic model of machine tools under the rigid-body assumption has been long studied [19, 20, 21, 5]. When nominal X, Y, and Z-positions 
are given by $p_{i}^{*}=\left[x_{i}^{*}, y_{i}^{*}, z_{i}^{*}\right] \in \mathbb{R}^{3}$, the actual TCP, $p_{i} \in \mathbb{R}^{3}$, is linearly related to geometric error parameters as follows:

$$
p_{i}=C_{\text {kinematic }, \mathrm{i}}\left(p_{i}^{*}\right) \cdot E_{i}\left(p_{i}^{*}\right)
$$

where

$$
E_{i}\left(p_{i}^{*}\right):=\left[E_{X}\left(x_{i}^{*}\right)^{T}, E_{Y}\left(y_{i}^{*}\right)^{T}, E_{Z}\left(z_{i}^{*}\right)^{T}, E_{C(0 X) Y}, E_{B(0 X) X}, E_{A(0 Y) Z}\right]^{T} \in \mathbb{R}^{21}
$$

$E_{X}\left(x_{i}^{*}\right) \in \mathbb{R}^{6}$ represent a set of geometric error parameters of X-axis at the position $X=x_{i}^{*}$, and is given by:

$$
E_{X}\left(x_{i}^{*}\right):=\left[E_{X X}\left(x_{i}^{*}\right), E_{Y X}\left(x_{i}^{*}\right), E_{Z X}\left(x_{i}^{*}\right), E_{A X}\left(x_{i}^{*}\right), E_{B X}\left(x_{i}^{*}\right), E_{C X}\left(x_{i}^{*}\right)\right]
$$

where $E_{X *}, E_{Y *}$, and $E_{Z *} \in \mathbb{R}$ are the linear error motions of the axis $*(*=\mathrm{X}, \mathrm{Y}, \mathrm{Z})$ in $\mathrm{X}, \mathrm{Y}$, and $\mathrm{Z}$ directions, respectively [2]. $E_{A *}, E_{B *}$, and $E_{C *} \in \mathbb{R}$ are its angular error motins around $\mathrm{X}, \mathrm{Y}$, and $\mathrm{Z}$ directions. $E_{C(0 X) Y}, E_{B(0 X) X}, E_{A(0 Y) Z} \in \mathbb{R}$ represent the squareness error of Y to X, Z to $\mathrm{X}$, and $\mathrm{Z}$ to $\mathrm{Y}$, respectively.

In Eq. (4), $C_{\text {kinematic }}\left(p_{i}^{*}\right) \in \mathbb{R}^{6 \times 21}$ represents the machine's kinematic model. Its formulation, dependent on the machine's configuration, can be found in many publications. For example, for the machine configuration shown in Fig. 5, the formulation can be found in [22].

For the given set of command positions, $p^{*}=\left\{p_{i}^{*}\right\}_{i=1, \cdots, N}$, Eq. (4) can be combined as:

$$
p=C_{\text {kinematic }}\left(p^{*}\right) \cdot E\left(p^{*}\right)
$$

where

$$
E\left(p^{*}\right)=\left[\left\{E_{X}\left(x_{i}^{*}\right)\right\}_{i=1 \sim N_{x}},\left\{E_{Y}\left(y_{j}^{*}\right)\right\}_{j=1 \sim N_{y}},\left\{E_{Z}\left(z_{k}^{*}\right)\right\}_{k=1 \sim N_{z}}, E_{C(0 X) Y}, E_{B(0 X) X}, E_{A(0 Y) Z}\right]^{T}
$$


Then, $X=\left[E\left(p^{*}\right),\left\{P_{j}\right\}_{j=1, \cdots, N_{t}},\left\{d_{0 j}\right\}_{j=1, \cdots, N_{t}}\right]$ can be identified by solving:

$$
\min _{X} \sum_{i=1 \cdots N, j=1 \cdots N_{t}}\left(f_{i j}\left(\left[\begin{array}{cc}
C_{\text {kinematic }}\left(p^{*}\right) & 0 \\
0 & I
\end{array}\right] X\right)-d_{i j}\right)^{2}
$$

where $I$ represents the unit matrix of proper size. The coordinate system constrains total 12 parameters in Eq. (8). Additionally, total 12 parameters must be constrained to be zero to avoid the redundancy in the definition of squareness errors and angular errors at the origin. The problem (9) can be locally solved by an iterative approach.

\section{Experiment}

\subsection{Experimental setup and procedure}

The objective of the experiment is to demonstrate direct and indirect measurement algorithms by using the tracking interferometer presented in Section 2. Its estimation accuracy will be experimentally investigated.

A commercial vertical-type five-axis machining center, NMV1500DCG by Mori Seiki Co., Ltd., of the configuration shown in Fig. 5, was tested. In this experiment, rotary axes are locked at $B=C=0^{\circ}$. Since only one tracking interferometer is currently available, the same measurement is repeated with the tracking interferometer set at four different positions on the machine table, assuming that the machine's unrepeatable positioning error is sufficiently small. By this reason, only the static (point-to-point) measurement of target position can be done; if simultaneous measurement can be done by using four or more tracking interferometers, its dynamic measurement is possible in principle. 
Figure 6 shows nominal target positions in $\bigcirc$ markers, as well as tracking interferometer positions (POS1 to 4). Within X100mm $\times$ Y100mm $\times$ Z100mm, there are total 43 nominal target positions with $20 \mathrm{~mm}$ step (in the XY plane). The measurement of 43 target positions took about 10 minutes. Figure 7 shows four tracking interferometer setups. In Figs. 7(a) and (c), it is fixed on a tilting table, such that the entire target path comes within the laser's reachable volume.

\subsection{Direct measurement of target positions}

Figure 8 shows laser displacement profiles measured from each tracking interferometer position. By applying the direct algorithm in Section 3, target positions are estimated. Figure 9 shows the estimated target position for each command position. An error between command and estimated target positions is magnified 1,000 times. Figure 9(a) shows the three-dimensional view, and Fig.9(b) shows its projection onto the XZ plane.

For comparison, this machine's two-dimensional positioning error was measured by using a two-dimensional digital scale (the term in [2]), or the cross grid encoder (KGM). It measures the position in two-dimensional orthogonal coordinate system by photoelectric scanning of a grid as the measurement reference [23]. KGM 182 by Heidenhain was used in this experiment. Figure 10 shows its setup. Figure 11 compares the projection of Fig. 9(a) onto the XY plane and the measured trajectory by using the KGM. The difference between two trajectories are within $3 \mu \mathrm{m}$. Note that the difference may be caused by the machine's thermal difference in two tests. 


\subsection{Indirect measurement of geometric error parameters}

By applying the indirect algorithm in Section 4, geometric error parameters are identified. Examples of estimated error motions, $E_{Y Y}$, the linear positioning error of Y-axis, and $E_{C Y}$, the yaw error motion of Y-axis, are shown in Fig. 12.

\section{Uncertainty analysis: issues in the present tracking interferom- eter prototype}

In the present experiment, the moving space of the target was limited within $100 \times 100 \times 100 \mathrm{~mm}$. This is mainly because the working range of two rotary axes of the present tracking interferometer is limited in $\pm 21^{\circ}$ (see Table 1). Although it is possible to modify tracking interferometer setups such that the laser beam's reachable space is enlarged, it likely increases the uncertainty in estimating the target position. To show this, the measurement uncertainty analysis will be presented in this section.

The uncertainly analysis for the multilateration-based measurement based on the Monte-Carlo simulation has been presented in past studies [9, 12, 16]. This paper adopts such a well-developed uncertainty assessment methodology to compare two sets of tracker positions shown in Table 3. Setup \#1 represents tracker positions in the experiment in Section 5. Setup \#2 represents the modified setup to enlarge the laser beam's reachable space. First, from the tracking interferometer's working ranges $\left( \pm 21^{\circ}\right)$, the laser beam's reachable space is calculated for both setups. In Fig. 13, painted dots represent target positions within the working range of all trackers. Clearly, Setup \#2 extends the laser beam's reachable space. 
The uncertainty in the length measurement by the laser interferometer is modelled as follows:

$$
u\left(d_{i j}\right)=u_{\mathrm{rand}}+u_{\mathrm{sys}} \cdot d_{i j}
$$

where $d_{i j}$ represents the laser displacement. $u_{\text {rand }}$ represents the unsystematic (random) uncertainty from the contributors such as the machine tool's unrepeatable positioning error or environmental fluctuation influencing the laser beam wave length. $u_{\text {sys }}$ represents the systematic uncertainty caused by e.g. the calibration error of the laser beam wave length. Since the main objective of the present analysis is the comparison of two setups, the detailed assessment of each contributor is not crucial (a part of the authors' previous works presented more detailed assessment of the prototype's uncertainty contributors[13]). Here, by evaluating e.g. the machine tool's accuracy tests or temperature fluctuation in the test site, $u_{\text {rand }}$ is modelled as a normallydistributed random number with the mean zero and the standard deviation $1 \mu \mathrm{m}$. $u_{\text {sys }}$ is modelled as a normally-distributed random number with the mean zero and the standard deviation $3 \mu \mathrm{m} / \mathrm{m}$.

From the Monte Carlo simulation [9, 12, 16], the standard uncertainty in each target position estimated by the direct algorithm in Section 3 is calculated. In Fig. 13, each dot's color represents the standard estimation uncertainty. In Setup \#1, a large portion of target positions is subject to the estimation uncertainty smaller than $3 \mu \mathrm{m}$. While Setup \#2 extends the measurable space significantly, the estimation uncertainty is larger in many positions.

This example suggests that it is difficult for the developed prototype to expand the measurable space while securing the estimation uncertainty below 
the required level.

\section{Uncertainty analysis: comparison of direct and indirect mea- surement algorithms}

The indirect algorithm, presented in Section 4, cannot evaluate the machine's volumetric error that is not in accordance with the kinematic model (4) - the applicability to unmodelled errors is a clear advantage of the direct algorithm presented in Section 3. On the other hand, the direct algorithm more likely causes larger estimation uncertainty for some portion of target positions. For the indirect measurement algorithm, an "averaging effect" with fitting to the model (4) often reduces the maximum uncertainty over the entire space. To illustrate this, this section compares the uncertainty analysis for both algorithms.

For clearer comparison, target positions in larger volume $(X=-1,500 \sim$ 1,500mm, $Y=-1,500 \sim 0 \mathrm{~mm}, Z=-700 \sim 0 \mathrm{~mm})$ is considered. Table 4 shows tracker positions. The same laser measurement uncertainty in Eq. (10) is assumed. First, the standard uncertainty in the estimation of each target position by the direct algorithm (Section 3) is calculated under this laser measurement uncertainty. Then, by applying the indirect algorithm (Section 4), geometric error parameters are identified under the same laser measurement uncertainty. Each target position is then calculated from the identified kinematic model (4). Figure 14 compares the standard uncertainty in each target position in both cases.

For the direct algorithm (Fig. 14(a)), while $86 \%$ of target positions is subject to the standard uncertainty smaller than $5 \mu \mathrm{m}$, there are some points 
with the uncertainty up to $15 \mu \mathrm{m}$. On the other hand, for the indirect algorithm (Fig. 14(b)), the estimation uncertainty is smaller than $5 \mu \mathrm{m}$ at all the points. This implies the "averaging effect" to reduce the maximum uncertainty over the entire workspace.

\section{Conclusion}

Based on the multilateration principle using the tracking interferometer, this paper presents the direct algorithm to calculate the three-dimensional position of the target, as well as the indirect algorithm to estimate geometric error parameters of the machine's kinematic model. Experiments showed that the developed tracking interferometer prototype estimated target positions with sufficient accuracy over $100 \times 100 \times 100 \mathrm{~mm}$. The measurable workspace is limited due to the limitation in the working range of two rotary axes of the prototype.

While the applicability to unmodelled errors is a clear advantage of the direct measurement algorithm, the indirect algorithm more likely reduces the maximum estimation uncertainty over the entire workspace due to an "averaging effect" with fitting to the model. This is illustrated by example uncertainty analysis.

\section{Acknowledgement}

The machining center used in the experiments is loaned from the Machine Tool Technologies Research Foundation (MTTRF) via Equipment on Loan Award Program. 


\section{References}

[1] ISO/CDTR 16907:2013, Numerical compensation of geometric errors of machine tools.

[2] ISO 230-1:2012, Test code for machine tools - Part 1: Geometric accuracy of machines operating under no-load or quasi-static conditions.

[3] Ramesh R, Mannan MA, Poo AN, Error compensation in machine tools - a review: Part I: geometric, cutting-force induced and fixturedependent errors, Int'l J. of Machine Tools and Manufacture 2000; 40(9); $1235-1256$.

[4] Schwenke H, Knapp W, Haitjema H, Weckenmann A, Schmitt R, Delbressine F, Geometric error measurement and compensation of machines -An update, CIRP Annals - Manufacturing Technology 2008, 57(2), $560-575$.

[5] Ibaraki S, Knapp W, Indirect Measurement of Volumetric Accuracy for Three-axis and Five-axis Machine Tools: A Review, International Journal of Automation Technology 2012; 6(2); 110-124.

[6] ISO 10360-2:2009 Geometrical product specifications (GPS) - Acceptance and reverification tests for coordinate measuring machines (CMM) - Part 2: CMMs used for measuring linear dimensions.

[7] Bringmann B, Küng A, Knapp W, A Measuring Artefact for true 3D Machine Testing and Calibration, CIRP Annals - Manufacturing Technology 2005, 54(1), 471-474. 
[8] Lau K, Hocken R, Haight W, Automatic laser tracking interferometer system for robot metrology, Precision Engineering 1986; 8(1); 3-8.

[9] Schwenke H, Franke M, Hannaford J, Kunzmann H, Error mapping of CMMs and machine tools by a single tracking interferometer, CIRP Annals - Manufacturing Technology 2005, 54(1), 475-478.

[10] Peggs GN, Virtual technologies for advanced manufacturing and metrology, Int'l J. of Computer Integrated Manufacturing 2003; 16(7/8).

[11] Hughes EB, Wilson A, Peggs GN, Design of a high-accuracy CMM based on multi-lateration techniques, CIRP Annals - Manufacturing Technology 2000; 49(1); 391-394.

[12] Schwenke H, Schmitt R, Jatzkowski P, Warmann C, On-the-fly calibration of linear and rotary axes of machine tools and CMMs using a tracking interferometer, CIRP Annals - Manufacturing Technology 2009; 58(1); 477-480.

[13] Yano T, Takatuji T, Osawa S, Motomura Y, Itabe T, Suzuki T, Development of a Spherical Motor Type Laser Tracker for the portable 3D Measurement System, Proc. of the International Symposium on Linear drives for Industrial Applications 2005; 254-257.

[14] Takatsuji T, Goto M, Kurosawa T, Tanimura Y, Koseki Y, The first measurement of a three-dimensional coordinate by use of a laser tracking interferometer system based on trilateration, Measurement Science and Technology 1998; 9; 38-41. 
[15] Takatsuji T, Goto M, Kirita A, Kurosawa T, Tanimura Y, The relationship between the measurement error and the arrangement of laser trackers in laser trilateration, Measurement Science and Technology 2000; $11(5) ; 477-483$.

[16] Umetsu K, Furutnani R, Osawa S, Takatsuji T, Kurosawa T, Geometric calibration of a coordinate measuring machine using a laser tracking system, Measurement Science and Technology 2005; 16; 2466-2472.

[17] Yano T, Actuator with Multi Degrees of Freedom, Next-Generation Actuators Leading Breakthroughs, Springer, 2010, 279-290.

[18] Takatsuji T, Goto M, Osawa S, Yin R, Kurosawa T, Whole-viewingangle cat's-eye retroreflector as a target of laser trackers, Measurement Science and Technology 1999; 10(7); 87-90.

[19] Soons J., Theuws F, Schellekens P, Modeling the errors of multi-axis machines: a general methodology, Precision Engineering 1992; 14(1); 5-19.

[20] Inasaki I., Kishinami K., Sakamoto S., Sugimura N., Takeuchi Y., Tanaka F., Shaper generation theory of machine tools - its basis and applications, Yokendo, Tokyo, 1997. (in Japanese)

[21] Abbaszadeh-Mir Y., Mayer J.R.R., Cloutier G., Fortin C., Theory and simulation for the identification of the link geometric errors for a five-axis machine tool using a telescoping magnetic ball-bar, Int'l J. of Production Research 2002; 40(18); 4781-4797. 
[22] Ibaraki S, Hata T, A new formulation of laser step diagonal measurement - Three-dimensional case, Precision Engineering 2010; 34(3); 516-525.

[23] Teimel A, Technology and applications of grating interferometers in high-precision measurement, Precision Engineering 1992; 14(3); 147-154.

[24] Ibaraki S, Measurement, control and compensation of volumetric errors of NC machine tools (technical article), Journal of the Society of Instrument and Control Engineers 2013; 52(1); 23-28 (in Japanese). 


\section{List of Tables}

1 Major specifications of the tracking interferometer. . . . . . 21

2 Major specifications of the cat's eye retroreflector (by Etalon

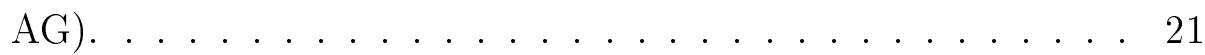

3 Tracking interferometer positions. . . . . . . . . . . . 22

4 Tracking interferometer positions. . . . . . . . . . . . 22

\section{List of Figures}

1 Tracking interferometer $[13,24] \ldots \ldots \ldots . \ldots . \ldots 23$

2 Principle of tracking. (a) When the shift of laser spot on the quadrant photo diode is detected, (b) the mirror is tilted by spherical motor to regulate the laser spot at the center of quadrant photo diode $[24] \ldots \ldots \ldots \ldots . \ldots . \ldots . . \ldots 23$

3 Controller configuration. . . . . . . . . . . . . 24

4 Configuration of multilateration-based measurement by four tracking interferometers. . . . . . . . . . . 25

5 Configuration of the machining center. . . . . . . . . 25

6 Tracking interferometer positions (POS1 to 4) and target trajectory $(\bigcirc$ markers $)[24] \ldots \ldots \ldots \ldots$

7 Tracker setups. (a) Tracker setup \#1 (POS1). (b) Tracker setup \#2 (POS2). (c) Tracker setup \#3 (POS3). (d) Tracker setup \#4 (POS4) . . . . . . . . . . . . . . 27

8 Measured laser displacement profiles from each tracking interferometer position (POS1 to 4). The horizontal axis represents the target position number. . . . . . . . . 28 
9 Estimated target positions. An error between command and estimated target positions is magnified 1,000 times. (a) In three-dimensional view. (b) Projection onto XZ plane. . . . . 29

10 Measurement setup by a cross grid encoder. . . . . . . . . . 30

11 Measured target positions by a cross grid encoder (KGM) in comparison with estimates by the tracking interferometer. Projection onto the XZ plane [24]. . . . . . . . . . . 30

12 Examples of estimated error motions. (a) $E_{Y Y}$, linear positioning error of Y-axis. (b) $E_{C Y}$, yaw error motion of Y-axis. . 31

13 The standard uncertainty in the estimation of target positions under laser interferometer measurement uncertainties. (a) Setup \#1. (b) ]Setup \#2. . . . . . . . . . . . . . . . . 32

14 The standard uncertainty in the estimation of target positions under laser interferometer measurement uncertainties. (a) Estimated by the direct algorithm. (b) Estimated by the indirect

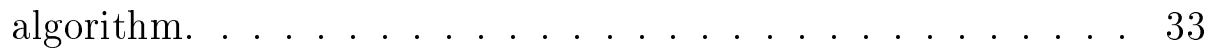


Table 1: Major specifications of the tracking interferometer.

\begin{tabular}{|c|c|}
\hline Size $^{1}$ & $88 \times 85 \times 71 \mathrm{~mm}$ \\
\hline Weight $^{1}$ & $0.75 \mathrm{~kg}$ \\
\hline Motor & A,B: AC servo spherical motors \\
\hline $\begin{array}{l}\text { Rotary encoder } \\
\text { resolution }\end{array}$ & $\begin{array}{l}\text { A, B: 20-bit } \\
\left(0.000343^{\circ} / \text { pulse }\right)\end{array}$ \\
\hline Stroke & $\mathrm{A}, \mathrm{B}: \pm 21^{\circ}$ \\
\hline $\begin{array}{l}\text { Angular positioning } \\
\text { resolution }\end{array}$ & $\begin{array}{l}\text { A: } 1.56 \text { mdeg } \\
\text { B:1.27 mdeg }\end{array}$ \\
\hline Torque & $\begin{array}{l}\text { A: } 0.20 \mathrm{Nm} \\
\text { B: } 0.03 \mathrm{Nm}\end{array}$ \\
\hline Max. speed & A, B: $90^{\circ} / \mathrm{sec}$ \\
\hline $\begin{array}{l}\text { Repeatability of angular } \\
\text { positioning (measured) }\end{array}$ & $\mathrm{A}, \mathrm{B}:< \pm 14 \mathrm{mdeg}$ \\
\hline Mirror diameter & $\phi 8 \mathrm{~mm}$ \\
\hline
\end{tabular}

Table 2: Major specifications of the cat's eye retroreflector (by Etalon AG).

\begin{tabular}{l|l}
\hline Viewing angle & $\pm 80^{\circ}$ \\
\hline Optical form deviation $^{1}$ & $<0.2 \mu \mathrm{m}$ \\
(circularity) & \\
\hline${ }^{1}:$ calibrated by the manufacturer.
\end{tabular}


Table 3: Tracking interferometer positions.

\begin{tabular}{l|l|l}
\hline & Setup \#1 & Setup \#2 \\
\hline POS1 & $(-76.0,-108.8,-52.2)$ & $(-5.0,-5.0,-234.0)$ \\
\hline POS2 & $(39.5,-6.7,-183.7)$ & $(105.4 .47 .8 .-234.0)$ \\
\hline POS3 & $(180.9,-100.7,-49.0)$ & $(64.5,81.1,-174.0)$ \\
\hline POS4 & $(212.4,83.1,-176.0)$ & $(38.5,20.6,-174.0)$ \\
\hline
\end{tabular}

Table 4: Tracking interferometer positions.

\begin{tabular}{l|l}
\hline POS1 & $(1000,-600,-300)$ \\
\hline POS2 & $(-1700,-1600,-1200)$ \\
\hline POS3 & $(100,-100,-600)$ \\
\hline POS4 & $(-1600,700,-900)$ \\
\hline
\end{tabular}




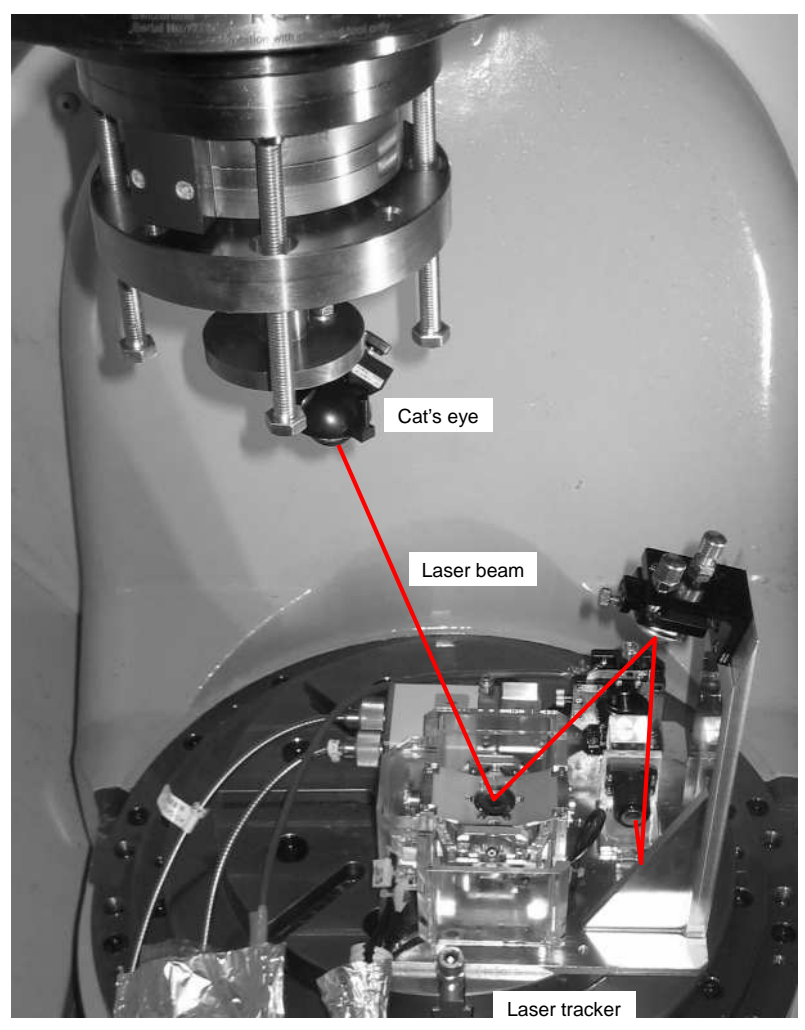

Figure 1: Tracking interferometer [13, 24].

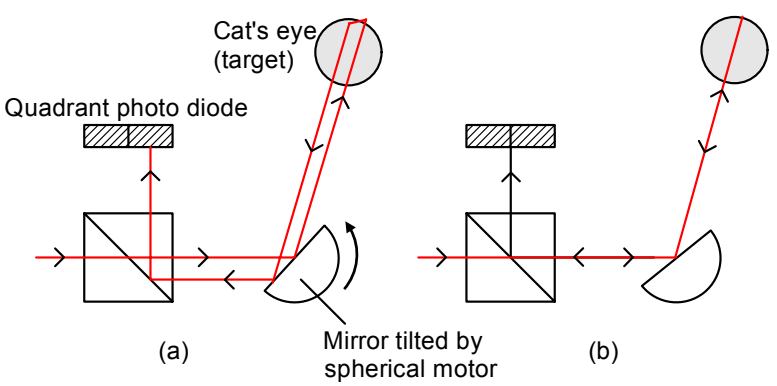

Figure 2: Principle of tracking. (a) When the shift of laser spot on the quadrant photo diode is detected, (b) the mirror is tilted by spherical motor to regulate the laser spot at the center of quadrant photo diode [24]. 


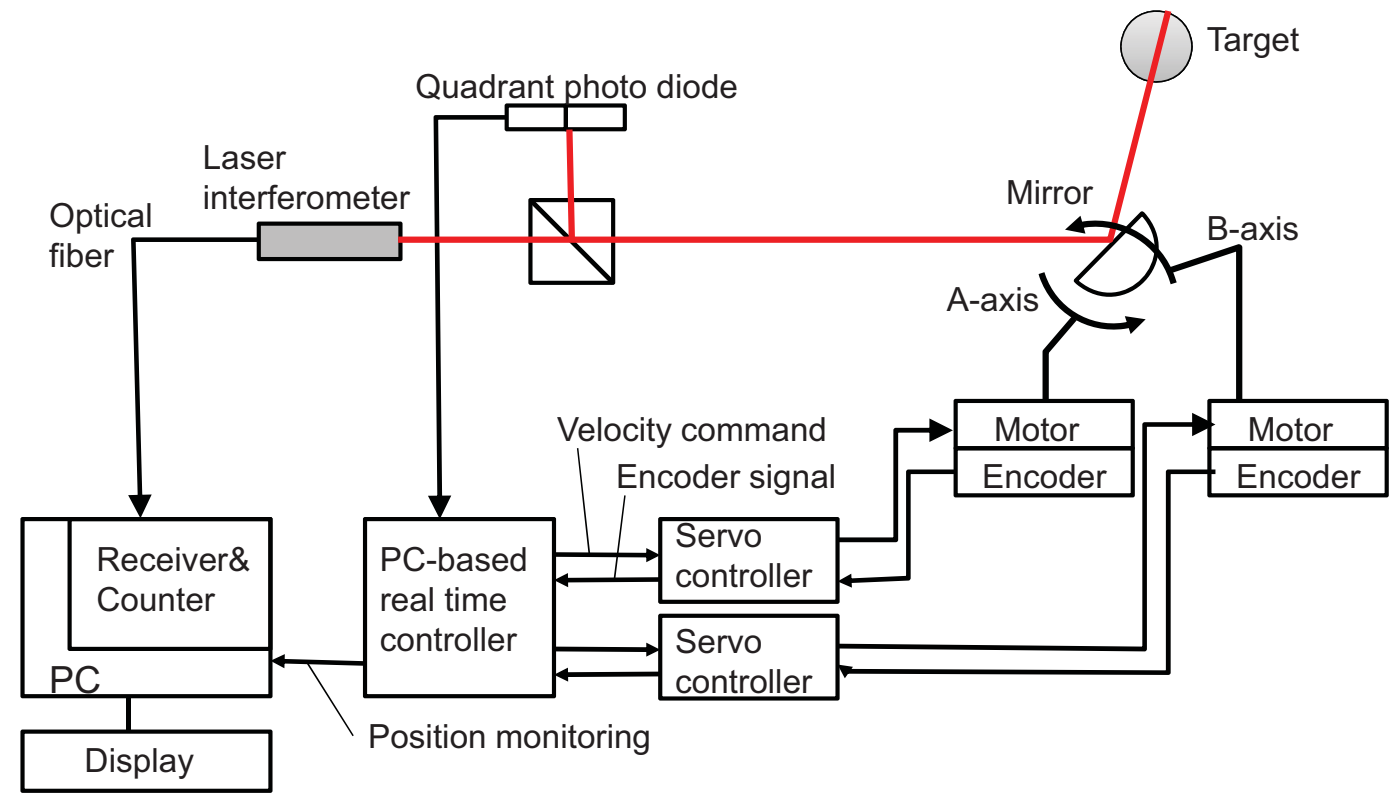

Figure 3: Controller configuration. 


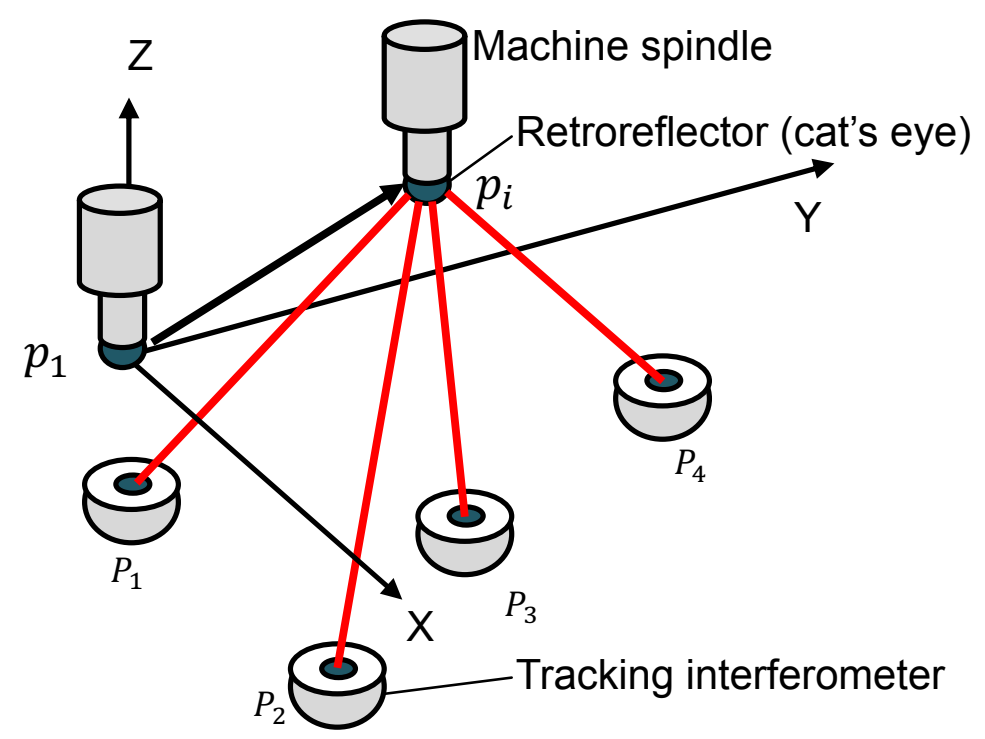

Figure 4: Configuration of multilateration-based measurement by four tracking interferometers.

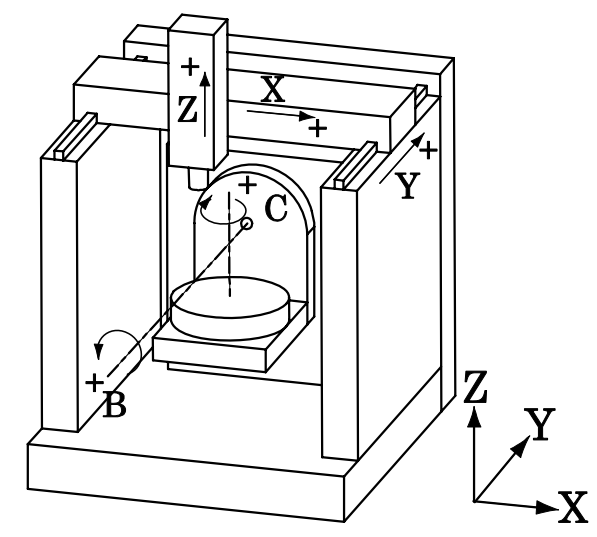

Figure 5: Configuration of the machining center. 


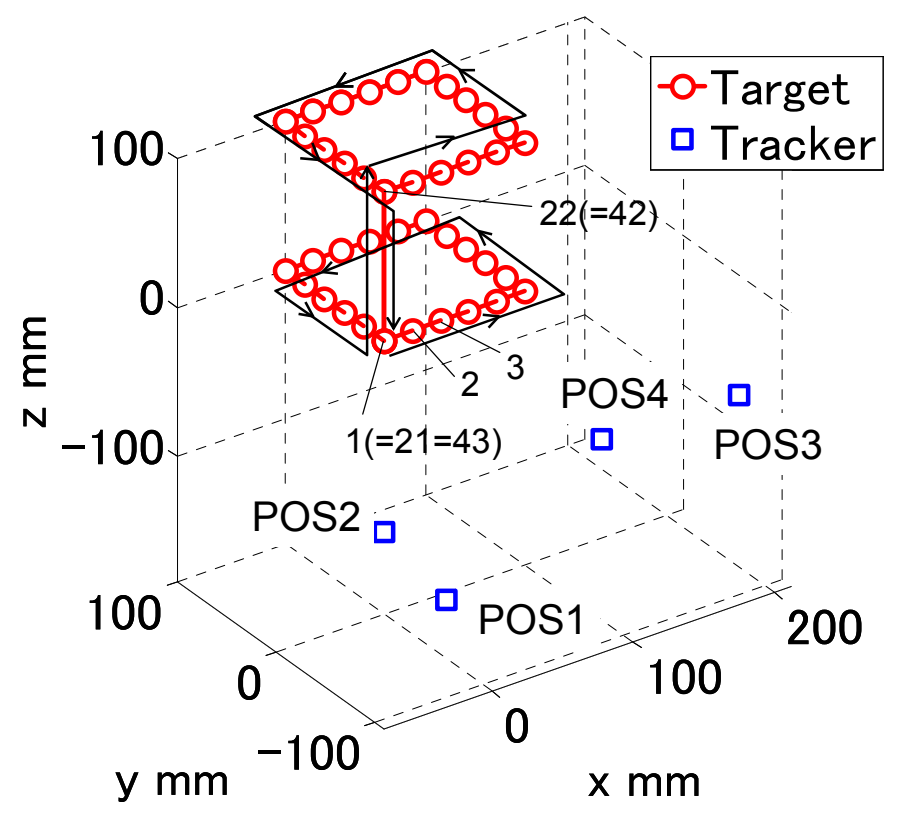

Figure 6: Tracking interferometer positions (POS1 to 4) and target trajectory ( $\bigcirc$ markers) [24]. 


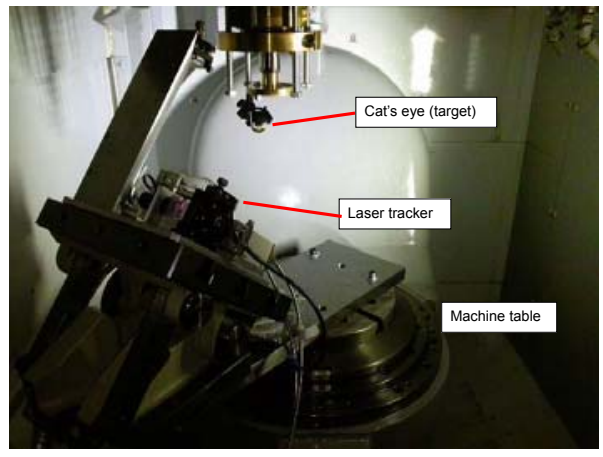

(a) Tracker setup \#1 (POS1)

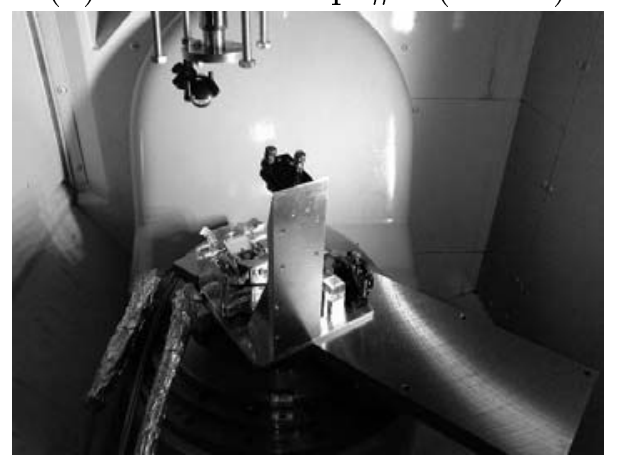

(b) Tracker setup \#2 (POS2)

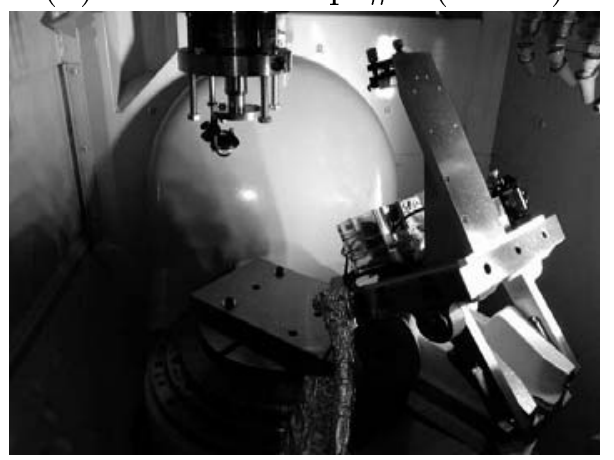

(c) Tracker setup \#3 (POS3)

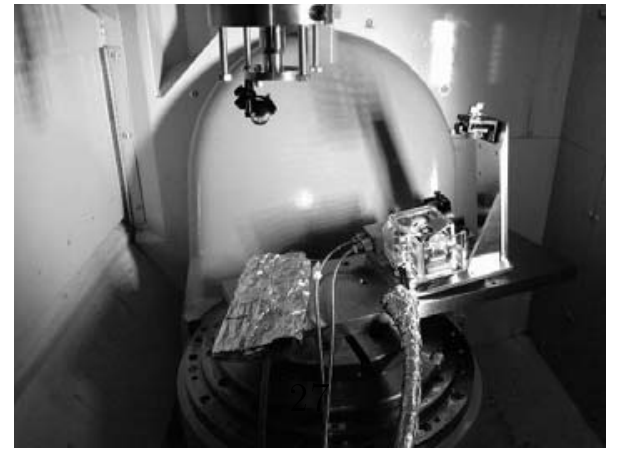

(d) Tracker setup \#4 (POS4)

Figure 7: Tracker setups. (a) Tracker setup \#1 (POS1). (b) Tracker setup \#2 (POS2).

(c) Tracker setup \#3 (POS3). (d) Tracker setup \#4 (POS4). 


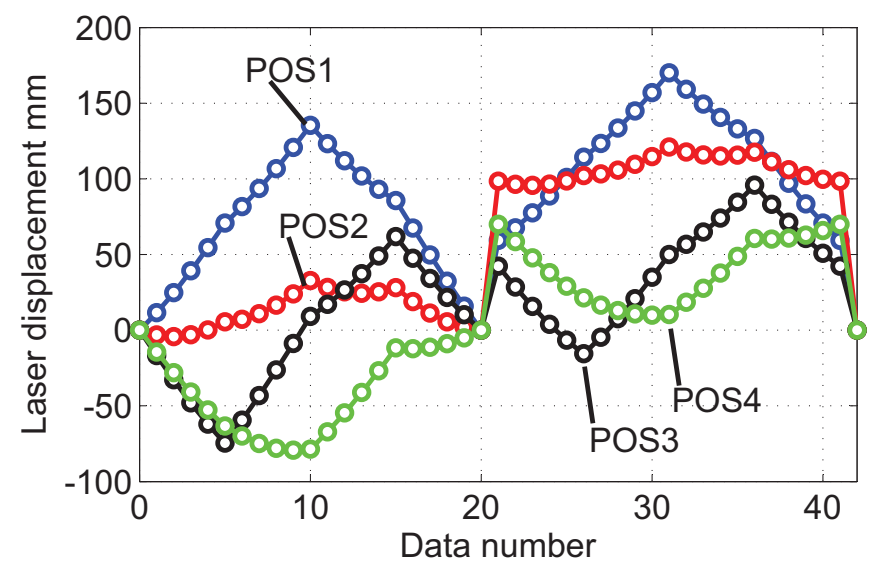

Figure 8: Measured laser displacement profiles from each tracking interferometer position (POS1 to 4). The horizontal axis represents the target position number. 


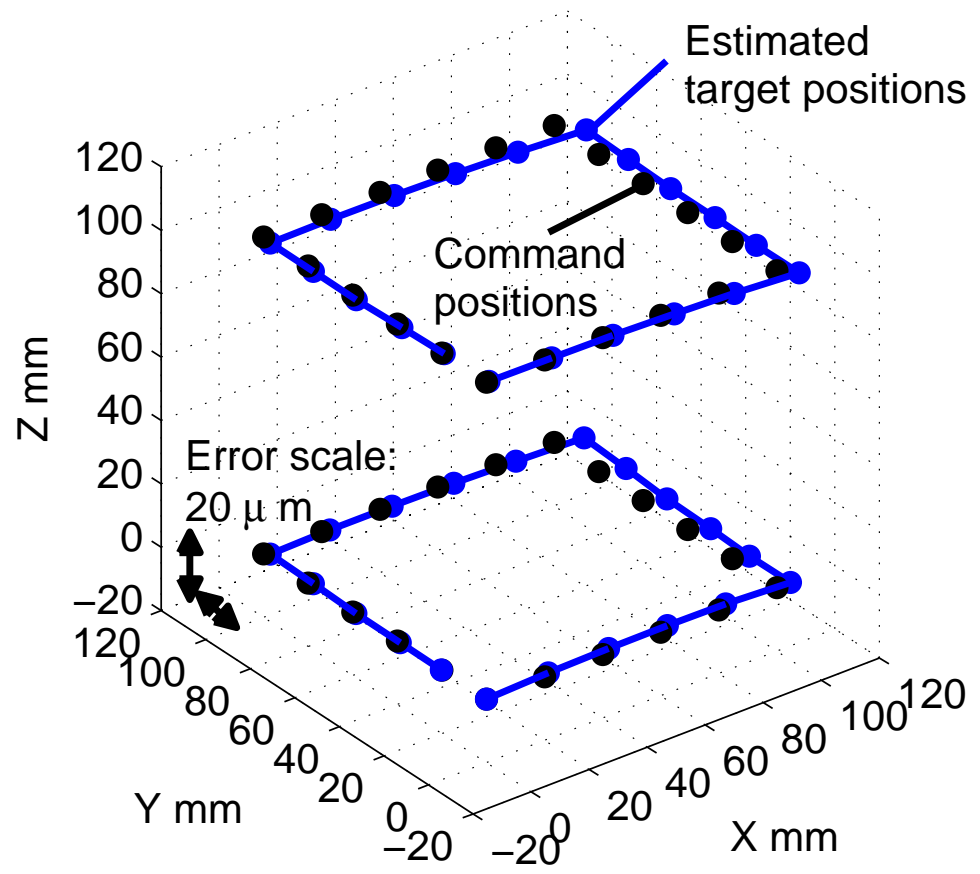

(a) In three-dimensional view [24].

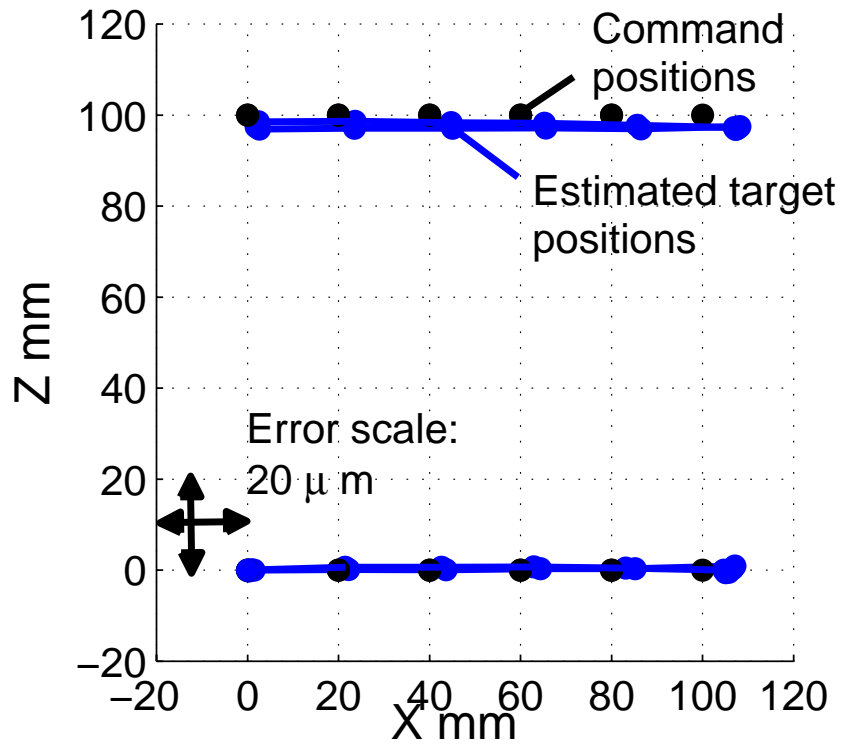

(b) Projection onto XZ plane.

Figure 9: Estimated target positions. An êror between command and estimated target positions is magnified 1,000 times. (a) In three-dimensional view. (b) Projection onto XZ plane. 


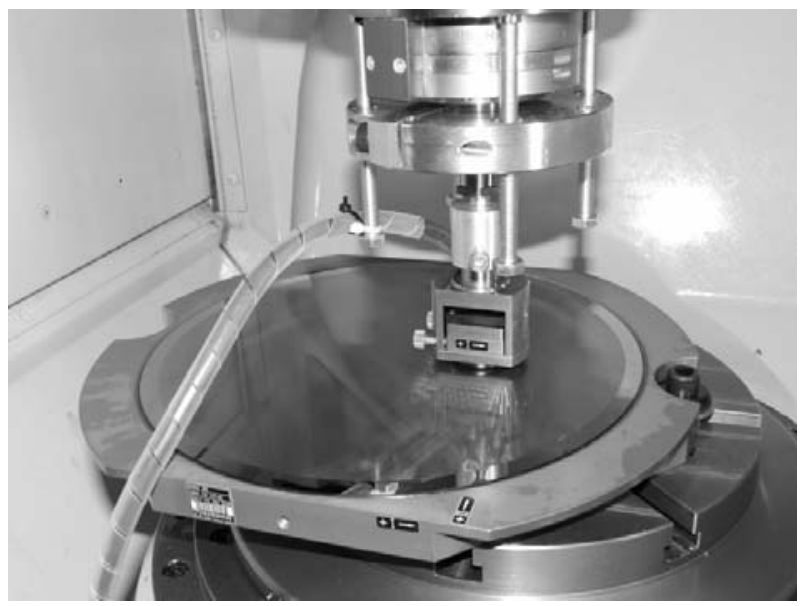

Figure 10: Measurement setup by a cross grid encoder.

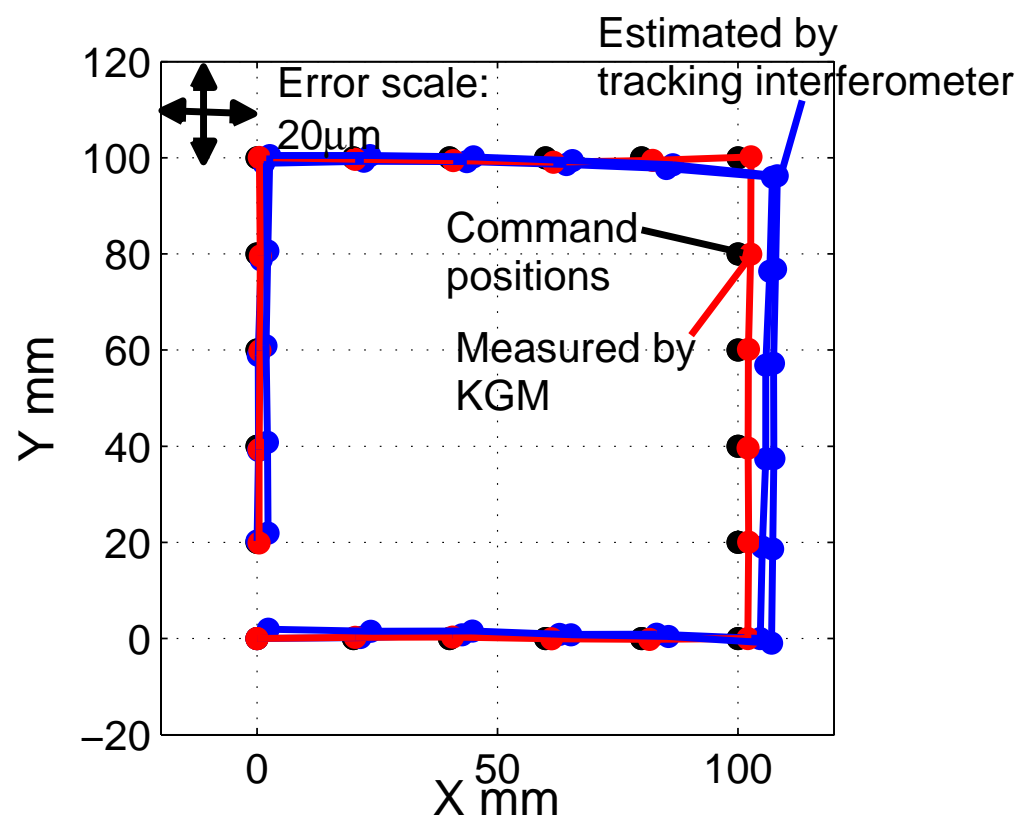

Figure 11: Measured target positions by a cross grid encoder (KGM) in comparison with estimates by the tracking interferometer. Projection onto the XZ plane [24]. 


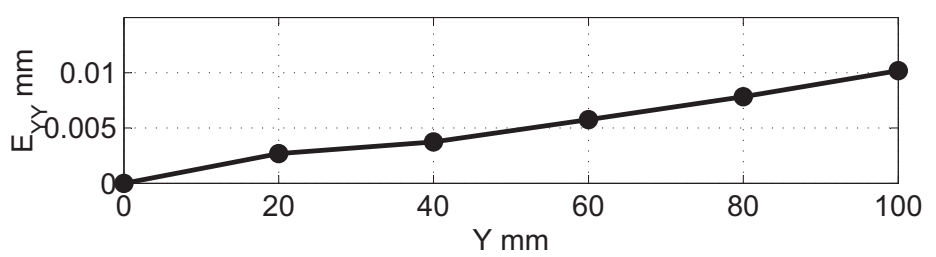

(a) $E_{Y Y}$, linear positioning error of $Y$-axis.

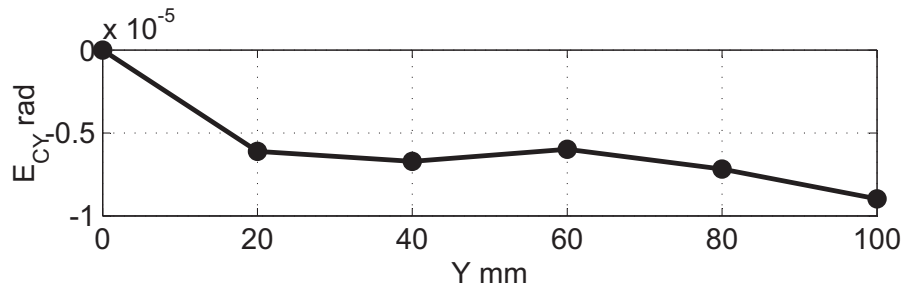

(b) $E_{C Y}$, yaw error motion of Y-axis.

Figure 12: Examples of estimated error motions. (a) $E_{Y Y}$, linear positioning error of Y-axis. (b) $E_{C Y}$, yaw error motion of Y-axis. 


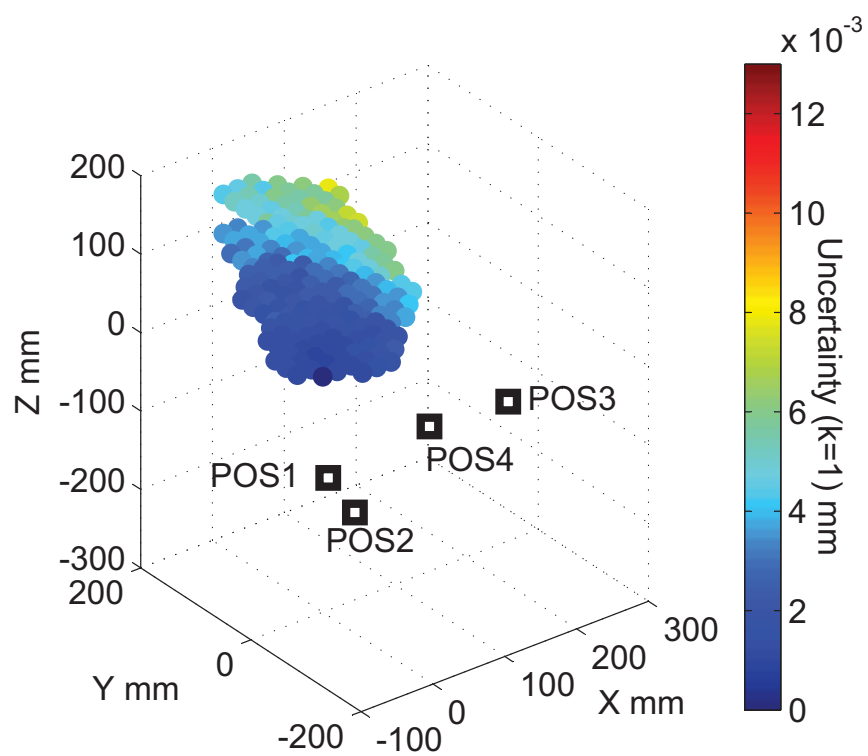

(a) Setup \#1

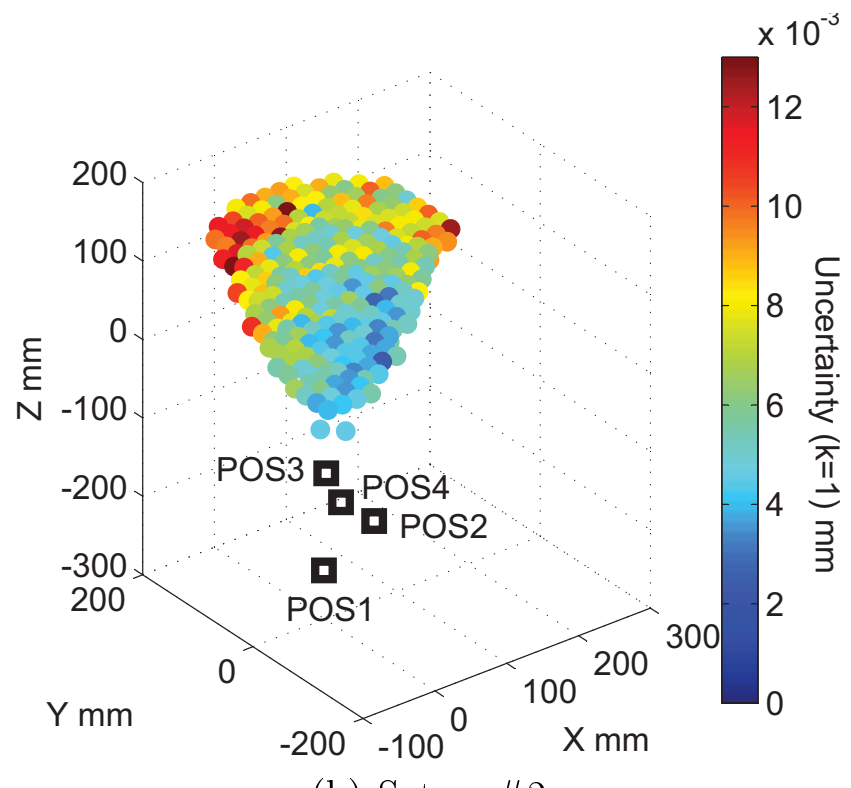

(b) Setup \#2

Figure 13: The standard uncertainty in the estimation of target positions under laser interferometer measurement uncertainties. (a) Setup \#1. (b) ]Setup \#2. 


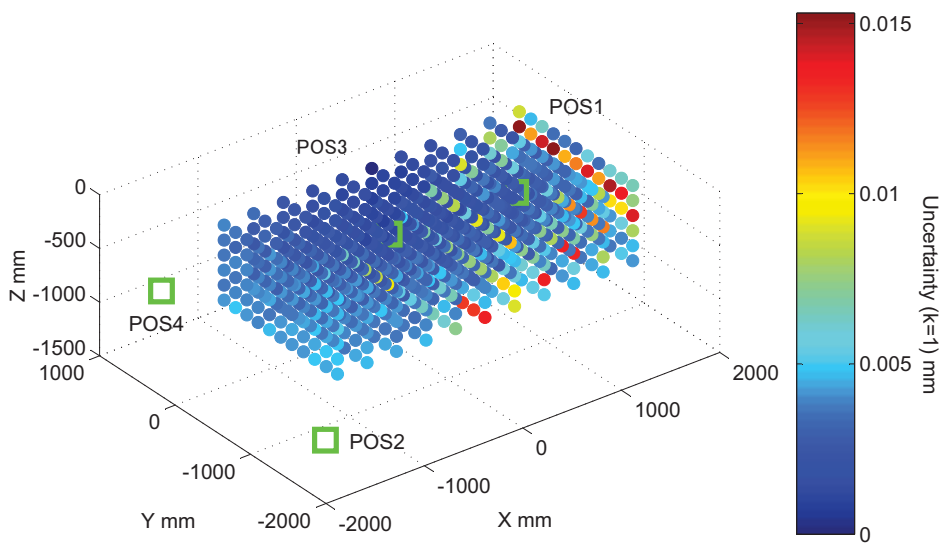

(a) Estimated by the direct algorithm.

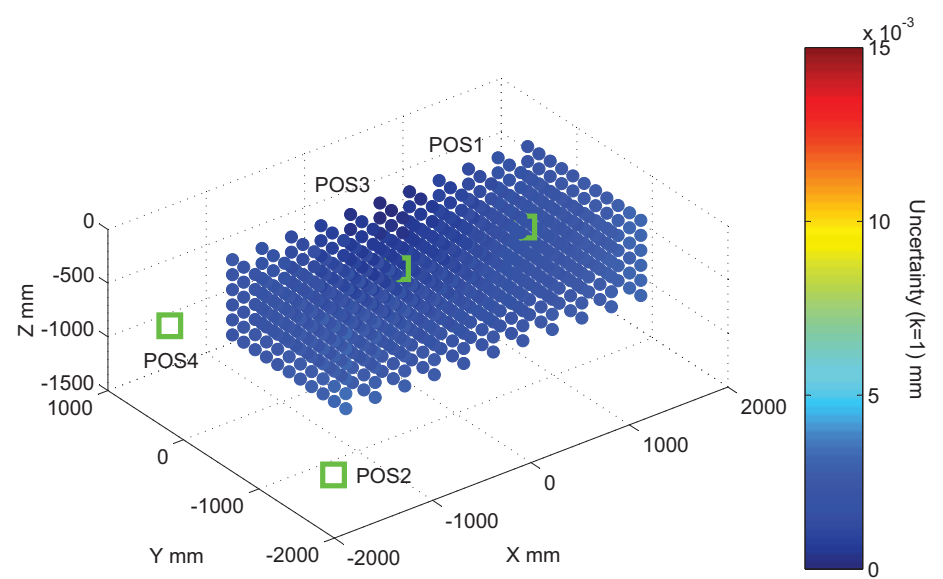

(b) Estimated by the indirect algorithm.

Figure 14: The standard uncertainty in the estimation of target positions under laser interferometer measurement uncertainties. (a) Estimated by the direct algorithm. (b) Estimated by the indirect algorithm. 